\title{
Age and gender differences in loneliness during the COVID-19: Analyses on large cross-sectional surveys and emotion diaries
}

1 Yoonwon Jung ${ }^{1}$, Yoon Kyung Lee ${ }^{1}$, Sowon Hahn ${ }^{1^{*}}$

$2{ }^{1}$ Department of Psychology, Seoul National University, Seoul, Republic of Korea

3 * Correspondence:

4 Sowon Hahn

5 swhahn@snu.ac.kr

6 Keywords: Loneliness, COVID-19, Age-Gender Interaction in Predicting Loneliness, Text Analysis,

7 Topic Modeling (LDA)

8 Abstract

The recent COVID-19 outbreak changed how age and gender affect loneliness and had a severe impact on the mental health of susceptible groups. This study investigated the joint influence of age and gender on loneliness, along with other psychological variables during the COVID-19 pandemic in South Korea.

We utilized large public data containing more than 4,000 self-reports and 20,000 emotion diaries from October 2020 to March 2021. Hierarchical regression, t-test, and analysis of variance were used to examine the effect of age, as a continuous or categorical variable, and gender on loneliness. We also utilized $\mathrm{N}$-gram frequency analyses and topic modeling to analyze the differences in expressions used by the age-gender groups in the diaries.

Analyses of surveys indicated that loneliness increased with age and that women were lonelier than men. However, age and gender interacted to predict loneliness: younger women and older men experience higher levels of loneliness. Age and age-gender interactions remained significant even after considering demographic and personality risk factors. Moreover, there were significant gender differences within the 20s and 30s. Furthermore, text analyses revealed that all age-gender groups, except women in their 60 s to $70 \mathrm{~s}$, frequently expressed anxiety and depression. Also, women in their 20 s expressed work experiences and difficulties in searching for a job, while women in their 30s highlighted childcare challenges and a lack of social connections. Lastly, spirituality was one of the major things expressed by women in their 50s to 70s, but not by the other groups.

This study confirmed the global trend of loneliness during COVID-19, while also illustrating cultural differences in loneliness trends that reflect social and psychological challenges in South Korea during the pandemic. Moreover, Text analyses provided potential explanations for the differences in loneliness by age and gender groups. Interventions targeting younger women and older men should be established. 
Age-Gender Differences in Loneliness

\section{$1 \quad$ Introduction}

Loneliness arises when an individual's level of social connection does not reach the desired level (1). It is the idiosyncratic feelings of isolation, not necessarily caused by objective social isolation, meaning that one can feel "lonely in the crowd" (1). Research on loneliness indicates that loneliness can cause serious psychological and physical health problems that needs to be addressed. Lonely individuals experience mental health problems, such as anxiety, depression, and low life satisfaction (2-5), as well as physiological problems, such as high blood pressure, coronary heart disease, and strokes $(6,7)$. Additionally, loneliness is associated with a $32 \%$ increase in the likelihood of mortality (8), which is comparable to the risk of heavy smoking (9). Risk factors that affect loneliness include marital status, physical health, socioeconomic status, quantity and quality of social relationship, and personality traits such as neuroticism and extraversion (10-13).

Prevalence and severity of loneliness may have been exacerbated during COVID-19 due to the decline of in-person social contact. Research on residents of the United States indicated elevated levels of loneliness during the last week of stay-at-home measures in April, 2020 (14), and during the first six months of lockdown (15). Moreover, nation-wide lockdowns increasingly led people to feel lonely in Europe $(16,17)$. However, a different stream of research suggested no significant increase in loneliness during the pandemic. During the two occasions in the U.S. where the social restriction measures were strictly enforced, there was no significant loneliness surge than before the pandemic (18). In addition, the prevalence of loneliness among adults in the UK remained the same before and during the pandemic $(19,20)$. Given that researchers raised concerns regarding loneliness reaching the level of an epidemic even before the coronavirus outbreak, loneliness levels may have remained stable during COVID-19 due to loneliness being already high before the pandemic (18, 21). Another possible explanation is that loneliness levels may have stabilized after the introduction of lockdown measures, considering that the general mental health during COVID-19 followed such paths.(22). However, it has been reported that older adults, quarantined individuals, and emergency workers were lonelier during the "stay-at-home" measures, indicating vulnerability of certain groups $(18,23)$. Moreover, a recent study reported that gender differences in loneliness have widened during the pandemic (17). This underlines the need to examine loneliness trends during the pandemic in more specific populations and compare these among groups parceled by risk factors influencing loneliness during the pandemic.

Age is an important risk factor for loneliness. A nonlinear U-shape has been reported in the relationship between age and loneliness $(11,20)$. The youngest and the oldest age groups generally showed a higher prevalence and intensity of loneliness than the middle age group $(12,24,25)$. In particular, a previous study revealed that adults under 25 years and above 80 years were the loneliest (11). The moderating effect of age on the impact of loneliness risk factors, such as employment status, relationship status, and household size can explain such differences (11). Recent studies conducted during COVID-19 have presented different age-loneliness relationship patterns. A general decreasing trend was identified, with the younger generation exhibiting the highest levels of loneliness (19, 26-28). However, when aggregated across age groups, middle-aged individuals were the loneliest (29). Similarly, a larger population of older adults aged over 70 years experienced increased loneliness (30), while adults in their 50s experienced even higher levels of loneliness (31).

It is important to consider gender differences in loneliness during the COVID-19 pandemic. It has been reported that the epidemic increased gender disparities in loneliness (17). Also, the patterns of these disparities have changed over time. On the one hand, earlier studies showed mixed findings, with some studies indicating no significant differences between gender and loneliness, while others 
reported that males were lonelier than females $(32,33)$. However, a few recent studies, including those conducted during the pandemic, have reported that women are more vulnerable to loneliness $(11,20,26,28,34)$.

Finally, the rising trend in emotion research used text analyses on diaries, interviews, or speeches reported a strong correlation between emotion-related linguistic markers and negative emotion measures (44-47). Naturalistic language containing individual writers' emotional experiences convey detailed information not fully captured by structural questionnaires. Word frequencies reveal attentional focus on oneself and the surrounding environment (48-50). Moreover, recent development in computerized intelligence to analyze narratives in an automated manner enables automatic topic extraction and classification from texts. Therefore, despite their limitations of being descriptive $(50,51)$, analyses of emotional narratives during COVID-19 offer insightful information on the varying attentional focus of life experiences, along with diverse emotional information, by demographic groups based on the influential risk factors of loneliness.

The present study aimed to investigate the joint influence of age and gender on loneliness during COVID-19 in South Korea. By utilizing text analyses in addition to scale-based response measures, were were able to gain rich information regarding culture and lifestyle factors that are related to age and gender in South Korea. This study hypothesized that gender would moderate the relationship between age and loneliness in both conditions, where age is a categorical and continuous variable. Specifying age as a categorical variable enables statistical tests across age groups and compares the results with those of other studies (20). Age as a continuous variable, without the scores being aggregated across age groups, is needed to uncover a general trend in age-loneliness relationships (11). 
Age-Gender Differences in Loneliness

\section{Materials and Methods}

\section{$125 \quad 2.1 \quad$ Dataset}

This study used the "COVID-19 Emotion Diary with Empathy and Theory-of-Mind Ground Truths Dataset" (52). It is a public dataset funded by the Korea Data Agency and managed by a psychology laboratory at Seoul National University as part of the Data Voucher Project. The dataset was created to construct empathetic artificial intelligence (AI) models to enhance the mental wellbeing of people during the pandemic. It contains self-reported demographic and psychological variables that may have been impacted by the pandemic, which includes loneliness. It also has 5 written emotional diaries per participant that contain emotions and thoughts regarding their experiences during the COVID-19. The dataset was collected via online crowdsourcing. Response qualities were controlled via twofold inspection, first by the quality control workers at the crowdsourcing platform and then by the psychology experts (52). Therefore, we concluded that this dataset is a valid, high-quality source for research on the characteristics of loneliness experiences during the pandemic. The list of variables used in this study is in Table 1.

The researchers obtained written consent from the participants, and the participants were informed that their responses will be part of an open dataset (52). The responses were collected anonymously, and the participants were asked not to include any personally identifiable information about themselves or others. The posterior twofold inspection process ensured that all diaries did not contain any such information. Lastly, the collection process did not include any experiments that inflicted direct manipulation on the human mind or body. Therefore, utilizing the dataset for our research is subject to exemption from institutional Review Board (IRB) approval, under the declaration of Helsinki (53). It also complies with the approval exempt condition stated in Article 15 Paragraph 2 of the Bioethics And Safety Act and Article 13 of its Enforcement Rules in Korea (54).

\subsection{Measures}

In this study, we used the following variables from the dataset: loneliness, extraversion, neuroticism, 'remote working or schooling,' and 'social distancing' measures.

\subsubsection{Loneliness}

Six-item version of Revised UCLA loneliness scale (ULS-6) was used to measure loneliness $(55,56)$. It uses a 4-point Likert scale from 1('never') to 4('often') to indicate how often each item is descriptive of oneself (33). It measures unidimensional loneliness that captures the essential characteristics of subjectively experienced loneliness, which are the feeling of isolation and withdrawal. Measures used in the dataset were based on the translated version of the full-scale Revised UCLA Loneliness Scale $(33,57)$. The reliability of the scale was 0.83 in the current study.

\subsubsection{Extraversion and Neuroticism}

Newcastle Personality Assessor (NPA) was used to measure extraversion and neuroticism among Big 5 Personalities (58). NPA is a brief personality measure consisting of 12 items that exhibited the highest correlations with the aggregated scores of the five-factor measure from the International Personality Item Pool. It uses a 5-point Likert scale from 1('strongly disagree') to 5 ('strongly agree') to indicate how much each item is descriptive of oneself. There were 2 items for both extraversion and neuroticism. Cronbach's alpha score is sensitive to the number of items and 
Age-Gender Differences in Loneliness

165

166

167

168

169

170

171

172

173

174

175

176

177

178

179

180

181

182

183

184

185

186

187

188

189

190

191

192

193

194

195

196

197

198

199

200

201

202

203

204

205

relies upon strict assumptions that are more susceptible for short scales to violate $(59,60)$. Therefore, some researchers advise adopting the Spearman-Brown corrected coefficients instead of Cronbach's alpha for two-item scales (60). In this study, we calculated both Cronbach's alpha and SpearmanBrown coefficients. Cronbach's alpha scores were 0.48 for extraversion, and 0.74 for neuroticism, and Spearman-Brown coefficients were 0.65 for extraversion and 0.85 for neuroticism in this study.

\subsubsection{Remote Working or Schooling, and Social Distancing}

A single-item scale was used to assess whether participants work remotely or receive noncontact education, and whether the participants are restricting social contacts to less than twice a week (52). Participants responded as either 'yes' or 'no'.

\subsection{Analysis}

We grouped the participants into 5 age ranges: 20s, 30s, 40s, 50s, and 60s-70s. Education and income levels were defined as continuous variables. The impact of living alone on loneliness was compared to the impact of other household types. Twelve responses of the 'other' household type were re-assigned into either 'living with immediate family,' or 'living with people other than immediate family' household types based on how the participants' additional descriptions of their specific situations. We relocated 10 responses to household 'living with immediate family,' and 2 responses to 'living with people other than immediate family.'

\subsubsection{Statistical Analysis}

Four thousand, three hundred fifty original responses were collected for five months (from October 2020 to March 2021). Among them, we removed missing data points and excluded responses from participants who reported their education level as 'other'. Also, individuals who wrote the number of people living together as 20 people or more without valid reasons (i.e. who were in the military service; all South Korean men should serve in the military and live in barracks while serving) were excluded. We finalized 4,017 responses in total eligible for analyses.

For analyses involving age as a categorical variable, we calculated mean loneliness ratings for each age-gender group, as well as 95 percent confidence intervals. We performed Welch two-sample t-test to test the statistical significance of gender differences within some age groups. To prevent the increased risk of type 3 error, we chose a planned comparison for additional analysis on the analysis of variance (ANOVA) results over post-hoc analysis. R 4.1.0 was used to conduct all statistical analyses.

For analyses on age as a continuous variable, we expected that a linear model would be appropriate for modeling loneliness, considering that participants' age ranges were from the 20s to 70 s and that the dataset was collected during the COVID-19 pandemic $(11,19,20,26)$. We conducted two hierarchical regression analyses: first to examine the influence of gender and age interaction on predicting loneliness, and then to test the final model obtained from the first regression analysis against the other risk factor for loneliness. Since the regression models contained interaction terms, we standardized all continuous variables to control Variance Inflation Factors (VIF) and increase the interpretability of coefficients in all models. To improve readability, we used the unstandardized version of the model for plotting graphs. Statistical significance was tested against $p<0.05$ as a two-tailed test. 


\subsubsection{Text Analysis}

207

208

209

210

211

212

213

214

215

216

217

218

To gain more insight into the gender differences in the relationship between age and loneliness, we conducted $\mathrm{N}$-gram frequency analyses and topic modeling with diary texts and compared the results by gender within the same age groups. We utilized texts from participants whose responses did not contain any null values to increase the size of text input as much as possible. We finalized 20,730 diaries as input for text mining models. We conducted all the analyses using python 3.8.

For N-gram frequency analyses, we calculated unigram and bigram frequencies as main analyses and calculated trigram frequencies when a deeper understanding of the contexts of target words was needed. $\mathrm{N}$-gram is a unit consisting of $\mathrm{N}$ number(s) of words that appear subsequently in a sentence. Unigram is a single word $(\mathrm{N}=1)$, and bigram is a continuous 2 words in a sentence $(\mathrm{N}=2)$. For example, in a sentence "Coronavirus made me stay home all day", "stay" and "home" are unigrams, and "stay home" and "home all" are bigrams.

To create $\mathrm{N}$-grams, we split the body texts of each diary into multiple sentences and each sentence into words. Then, we removed all punctuations, selected words with part-of speech (POS) tagging corresponding to nouns, and formed N-grams. N-grams that contained 'stop words' were not used. Stop words are words that commonly appear in a corpus but do not contain any meaningful information regarding the purpose of analyses. Finally, counts and frequencies on unigrams and bigrams that appear most frequently were calculated, mostly up to rank 30 .

We used Latent Dirichlet Allocation (LDA) for topic modeling. Topic modeling is a machine learning technique that derives latent topics from a large corpus consisting of multiple documents. One of the most popular topic models, Latent Dirichlet Allocation (LDA), models the distribution of latent topics in documents using Dirichlet distribution to determine the best topic sets (61). The model implementation was based on Collapsed Gibbs Sampling method $(62,63)$. The text preprocessing procedure was similar to that used when constructing $\mathrm{N}$-grams, with the exception that the stop words were removed before modeling. The vectorized token was created from the final form of processed tokens and used as input for modeling.

We ran coherence tests for each gender-age group model, altering two hyperparameters: the number of topics to be extracted, and the number of most frequent tokens to be deleted, to find the best model. We ran 300 iterations for each model. We chose the final model with the highest intertopic discriminability and topic interpretability among the three models with the highest coherence scores.

\section{Results}

\subsection{Sample Characteristics}

The participants' average age was $34.87(S D=12.85)$, with a range of 20 to 79 . There were 3,053 female workers, accounting for $76 \%$ of all participants. The average income level was 3.73 $(S D=2.510)$, and the average education level was $3.44(S D=0.97)$. The average loneliness score was $2.27(S D=0.71)$. Full sample characteristics by the age groups and gender are presented in Supplementary Tables 1 and 2. 
Age-Gender Differences in Loneliness

\subsection{Loneliness by Age and Gender: Age As a Continuous Variable}

We tested the hypothesis of gender moderating the relationship between age and loneliness in the context of age as a continuous variable (Table 2). Model $1(F(1,4015)=13.12, p<0.001)$ and model $2(F(2,4013)=15.13, p<0.001)$ were both significant. Model 2 showed that age $(t(4013)=2.03$, $p=0.043)$ and gender $(t(4013)=-5.24, p<0.001)$ were both significant, with women and older people being lonelier. The interaction effect between gender and age $(t(4013)=2.92, p=0.004)$ was also significant, with male participants showing greater variation to age in loneliness than female participants. Visualization of the effect of age on loneliness by gender group revealed that males were lonelier than females after reaching the age of 60 (Figure 1). According to hierarchical regression on models 1 and 2, gender and its interaction with age significantly increased the model's explanatory power $(F(2,4013)=17.33, p<0.001)$.

We conducted another hierarchical regression analysis with the final model from Table 2 as the baseline model (Table 3). We investigated whether gender and age remained as a significant predictor of loneliness in the presence of other risk factors for loneliness. In the first step, we added the following demographic variables to model 1 as control variables: income, family type, remote working or schooling, and social distancing. The resulting model (model 2) was statistically significant $(F(10,4006)=15.02, p<0.001)$, and the age, gender, and their interaction effect remained significant after demographic variables were included. The influence of age grew stronger, while the influence of gender weakened. Then, in the second step, we added the personality variables to form model 3.

With personality risk factors being added, model 3 was statistically significant $(F(12,4004)=116.60, p<0.001)$. The effect of age as well as the interaction between age and gender remained significant, whereas the effect of gender did not. However, we discovered that the correlation between age and neuroticism was stronger than the correlations between the other two variables, which may influence the significance of age and age-gender coefficients. We conducted an additional regression analysis of age, neuroticism, and age-neuroticism interaction (Supplementary Table 3). In predicting loneliness, neuroticism had a significant interaction effect with age as a continuous variable $(t(4013)=2.06, p=0.039)$. Age had a greater impact on predicting loneliness in participants with higher neuroticism scores. We, therefore, added age-neuroticism interaction term to model 3 to create model 4 , and model 4 was chosen as the final model. The model was statistically significant $(F(13,4003)=108.10, p<0.001)$, and explained $25.74 \%$ of loneliness variance overall. Age was a significant predictor of loneliness $(p<0.001)$, with males having larger age prediction coefficients in loneliness than females $(p=0.030)$. Lower income $(p=0.002)$, social distancing $(p<0.001)$, living alone compared to living with other people $(p=0.016)$, lower extraversion $(p<0.001)$, and higher neuroticism $(p<0.001)$ were also significant predictors of loneliness.

\subsection{Loneliness by the Age Groups and Gender: Age As a Categorical Variable}

We visualized the aggregated loneliness scores across age groups and gender. The general trend was that participants in their 40s, regardless of gender, reported a high level of loneliness. Among all age-gender groups, females in their 30s and 40s were the loneliest. We conducted Welch two-sample t-test on age groups with high gender differences (Figure 2). Female participants in 20s ( $M: 2.23,95 \%$ CI: $[2.20,2.26])$ and 30s (M: 2.45, 95\% CI: [2.390, 2.499]) were lonelier on average than male participants in 20s ( $M: 2.06,95 \% C I:[1.99,2.13])$ and 30s $(M: 2.16,95 \%$ CI: [2.07, 2.25]) each. Such gender differences in $20 \mathrm{~s}(t(531)=4.13, \mathrm{p}<0.001)$ and $30 \mathrm{~s}(t(423.59)=5.32, p<0.001)$ were both 
statistically significant. Although male participants in 60s-70s ( $M: 2.39, C I:[2.25,2.53])$ were lonelier on average than females in the same age group $(M: 2.25,95 \% C I:[2.11,2.38])$, such differences were not statistically significant.

We used ANOVA to evaluate the moderation effect of gender on the link between age as a continuous variable and loneliness. The main effect of gender $(F(1,4007)=2.70, p=0.019)$, age group $(F(4,4007)=23.20, p<0.001)$, and the interaction between gender and age groups $(F(4,4007)=10.20$, $p<0.001$ ) were all statistically significant (Table 4).

We also conducted a planned comparison to further test our assumptions about the loneliness levels in certain gender and age groups. Contrasts were constructed based on previous literature. We predicted that females would be lonelier than males. For contrasts within age and gender group levels, we predicted that individuals in their 20s to 30 s would be lonelier than those in their 40 s to $70 \mathrm{~s}$, and individuals in their 60s-70s would be lonelier than those in their 30s to 50s. Also, we speculated that respondents in their 20s would be lonelier than those in their 30 s, and respondents in their 40s would be lonelier than those in their 50s.

Results showed that females were lonelier than males $(t(4007)=2.35, p=0.019)$. Also, participants in their 20 s to 30 s were significantly less lonely than those in 40 s to $70 \mathrm{~s}(t(4007)=-3.83$, $p<0.001)$. Respondents in their 20s were lonelier than those in their 30s $(t(4007)=-4.69, p<0.001)$, and respondents in their 40s were lonelier than those in their 50s $(t(4007)=2.66, p=0.008)$. However, interaction effects revealed that female participants in their 20s to 30s were significantly lonelier than those in 40 s to 70 s while male participants in their 20s to 30s were significantly less lonely $(t(4007)=4.19, p<0.001)$. The full results for planned comparison are in Table 5.

\subsection{Frequent N-grams Used Within the Age-Gender Groups}

We investigated gender differences in the N-gram usage among participants in their 20s to 30s

(Tables 6 and 7). 'Child' was the 14th most frequent unigram for female participants with 0.48 word frequency, while the same word was 56th most frequent word for male participants with 0.00 word frequency. 'Delivery food' was the 24th most frequent bigram for females while it was 33rd for males, with bigram frequencies of 0.07 and 0.00 , respectively. The findings shouwed that females in their 20 s30 s were more concerned with child care and food provision than males in their 20s-30s during the COVID-19.

Another major gender difference in $\mathrm{N}$-gram usage among participants in their 20s to 30s was the frequency using the word 'confirmation'. 'Confirmation' was the 16th most frequent unigram used among females while being the 24th most frequent word among males, with word frequencies being 0.46 and 0.34 respectively. Moreover, 'All day long' was the 16th most frequently used bigram among females, with 0.09 bigram frequencies while it was the 29th among males, with 0.06 bigram frequencies. The results revealed that females in their 20-30s were more concerned about the virus and talked more about their experiences that lasted all day.

Another significant gender difference in N-gram usage among participants in their 20s to 30s was the use of 'traveling'. It was the $23^{\text {rd }}$ common bigrams used by females, while it was the $44^{\text {th }}$ common unigram used by males, with word frequencies being 0.46 and 0.34 respectively. We examined trigrams containing the word 'traveling' used by females in their 20s and 30s to reveal the context of the word being used. Frequent trigrams were 'traveling, because of, COVID-19', 'foreign, 
333

334

335

336

337

338

339

340

341

342

343

344

345

346

347

348

349

350

351

352

353

354

355

356

357

358

359

360

361

362

363

364

365

366

367

368

369

370

371

372

373

374 domestic, traveling', 'trip, planning, traveling', 'traveling, domestic, trip', 'COVID-19, situation, traveling', 'domestic, traveling, people', 'friend, planning, traveling', 'wedding, honeymoon, traveling', and 'honeymoon, traveling, canceled', 'eventually, traveling, canceled'. This revealed that 'traveling' was used in relation to their plans on traveling, people traveling during the COVID-19, and canceling traveling. We also looked at gender differences in the word usage among participants in their 60s-70s, but no major differences were found.

\subsection{Frequent Topics Discussed Within the Age-Gender Groups}

To further investigate the differences among age-gender groups, we used topic modeling on 10 specific age-gender groups and extracted the topics that are mainly discussed within each group (Supplementary Table 4). First, all females except in their 30s showed topics related to their pets (i.e., 'dog' or 'cat'), but no males did (Table 8). Moreover, all females had mother-related topics in each age group (i.e., 'mom') (Table 9). Specifically, females in their 30s frequently talked about one's domestic role (i.e. as a mom or wife) or about other mothers, whereas males in their 30s did not. We conducted additional bigram and trigram frequency analyses that contained 'mom' in the sequence of two or three words to examine context of the word in diary texts. We used token ' $<$ name $>$ ' to collectively replace unigrams representing the names of other people. The results revealed that females over 30s used 'mom' to refer to their mother (i.e., 'think, mom' 'my, mom, father', and 'distance, parent's home, mom'), other people (i.e., 'child, friend, mom', 'friend, mom', and '<name>, mom'), or themselves (i.e., 'child, mom, child', 'son, child, mom', 'mask, child, mom', and 'child, kindergarten, mom'). However, all males across age groups did not show topics with the word 'father' as a topic word, and the word 'wife' was used to refer to their marriage partner.

With topic modeling, we also discovered patterns in diaries written by females in their 20s and over 50. Females in their 20s wrote more about jobs and working than males in their 20s, and this pattern did not occur in the other age groups (Table 10). Two of the three work-related topics of females in their 20s were about thoughts and feelings about acquiring jobs. They included topic words describing internal states. The other was about non-contact interview or working experience, and it featured topic words that described situational elements. Meanwhile, females in their 50s and 60s-70s had topics regarding their spiritual life, as indicated by the topic words 'church' and 'worship' (Table 11), while males did not. Lastly, all age-gender groups, with the exception of females in their 60s-70s showed topics related to negative affect state, which included topic words like 'depression', 'stress', and 'annoyance'.

\section{Discussion}

\subsection{Analyses on the Effect of Age, Gender, and Other Variables on Loneliness Confirmed the Global Loneliness Trend While Suggesting Cultural Differences}

The regression model found a positive correlation between age and loneliness, which remained significant even after adding other risk factors. This finding contradicts recent studies conducted during the pandemic $(19,26)$, which revealed that age, as a continuous variable, had a negative association with loneliness. Considering that similar large-scale loneliness studies were conducted within Western contexts, the novelty may be due to cultural differences. Meanwhile, ANOVA results revealed that South Koreans in their 20s and 30s were lonelier, on average, than 
Age-Gender Differences in Loneliness

375

376

377

378

379

380

381

382

383

384

385

386

387

388

389

390

391

392

393

394

395

396

397

398

399

400

401

402

403

404

405

406

407

408

409

410

411

412

413

414

415

416

417 those in their 40-70s. The disparity in the age-loneliness relationship between the two analyses results indicates that the age-loneliness relationship can vary greatly depending on how the age variable is defined. Additionally, participants in their 40s reported higher levels of loneliness than those in the other age groups. This result corresponds with previous research, which indicated that the middle-aged group was the loneliest in the United Kingdom (29). In terms of the effect of gender on loneliness, women were generally lonelier than men, which is consistent with a recent study (11, $20,26,34)$.

However, the characteristics of the prevalence of loneliness could not be fully captured when age or gender was considered alone or separately. Regression analysis of the influence of age and gender on loneliness revealed that while women were lonelier than men on average, they exhibited a gradual increase in loneliness by age than men. Women were lonelier than men until 60 years, but men were lonelier than women after 60 . The age-gender interaction remained significant even when other risk factors were included. The ANOVA results indicated that while women in their 20s and 30 s were lonelier than those in their 40s, male participants showed the opposite trend. This suggests the importance of age and gender interaction in determining the severity of loneliness and that the interplay should thus be considered when identifying high loneliness groups.

\subsection{Text Analyses Results Provided Potential Explanations for the Differences in Loneliness by Age and Gender}

In South Korea, participants in their 20s and 30s showed the greatest gender differences in loneliness, with women being lonelier than men. Women in their 20s wrote more about their work experience than men and expressed a depressed state toward getting jobs. According to Statistics Korea, (64) the employment rate of Koreans in their 20s decreased by 4.2pp (percentage points) in January 2021 compared to the previous year, the steepest decrease among all age groups. It is likely that women suffered more from job loss in Korea during the pandemic, given that the increase in the number of unemployed women, 48.4pp, were higher than those of men, 25.9pp, after a year-long COVID-19 outbreak. The previous finding-employment status is a strong predictor of loneliness for adults under the age of 30-strengthens the argument that job difficulties may be a possible reason for younger women's increased levels of loneliness in South Korea (11).

South Korean women in their 30s wrote more about their domestic role (i.e. as a mother or wife) of caring for their families or about other mothers, while males in their 30s did not. A closer examination of diaries containing "child" or "mom" written by women in their 30 s indicated that women who were married and had children faced difficulties in supporting their children's non-contact education while also feeding their families (Table 12). Remote work, remote education, and reduced one-on-one meetups prompted by COVID-19 exacerbated loneliness and depression in mothers. This result is consistent with earlier studies: women were burdened with more household duties and parenting responsibilities during the COVID-19 pandemic $(65,66)$, Additionally, women who were pregnant or in the perinatal period experienced worsening mental health after the COVID-19 outbreak $(67,68)$.

The impact of motherhood on the mental health of South Korean women may not be limited to married women with children. Some diary inputs written by women in their 30s suggested they were prone to feeling lonely due to COVID-19 that prohibited physical contact with friends (Table 12). The majority of research on women's psychological well-being during the COVID-19 pandemic focused 
on married women about to or already raising their children. Younger women need to be given broader attention when designing interventions to address loneliness during COVID-19.

Some research conducted prior to COVID-19 identified the female gender as a risk factor, irrespective of age $(20,34)$. This contradicts this study's findings, which revealed that older women were less lonely than older men. Given that loneliness is associated with depression $(2,69)$ and lonely individuals demonstrated linguistic markers of depression and anxiety in self-written texts (70), this inclination is supported by the linguistic markers of emotions associated with loneliness - depression and anxiety - being less prevalent in diaries written by older women. Moreover, text analyses on diaries revealed that women in their 50s to 70s had spirituality-related interests. Older age and female gender have been reported to positively predict spirituality; and spirituality is positively correlated with subjective well-being in older adults (71-74). Such findings may provide an indirect explanation for why South Korean women are less lonely than men after the age of 60 .

\subsection{Contributions and Limitations}

This study utilized large-sized survey data collected using the validated measures of loneliness along with diaries collected from South Korean samples during COVID-19. No research on Korean people's loneliness levels has been conducted in this size, specifically during the COVID-19 pandemic. Gender-age groups in South Korea suffering from elevated levels of loneliness during the pandemic were identified by examining loneliness trends using the interaction between gender and age during the pandemic. Based on the text analyses, further explanations for gender differences in loneliness levels within age groups were provided. Based on this study's findings, possible interventions targeting loneliness-suffering populations in South Korea can be designed.

The sample size varied greatly across age groups and genders; more than half the participants were women in their 20s. Owing to the nature of online crowdsourcing, the characteristics of individuals who actively participated on such platforms may have affected the results (75). Future studies should expand their sample to include more information from older age groups and male gender groups. Furthermore, possible results of recruiting participants online should be considered. Moreover, future studies should investigate cultural differences in loneliness trends with cross-cultural results. Finally, future research should explore the differences in loneliness levels before and after the pandemic and address changes in individual levels of loneliness over time.

\section{$5 \quad$ Conflict of Interest}

The authors declare that the research was conducted in the absence of any commercial or financial relationships that could be construed as a potential conflict of interest.

\section{Author Contributions}

All authors contributed to the conception and design of the study. YJ performed the statistical analysis and wrote the first draft of the manuscript. All authors contributed to the manuscript revision, and read and approved the final manuscript. 
$457 \quad 7 \quad$ Funding

458 The authors received no specific funding for this work.

459

4608 Acknowledgments

461 This manuscript previously appeared online as a preprint, in https://doi.org/10.31234/osf.io/2bdek

462 (76).

463

4649 Data Availability Statements

465 A sample of "COVID-19 Emotion Diary with Empathy and Theory-of-Mind Ground Truths Dataset" 466 used for this study can be found at https://github.com/humanfactorspsych/covid19-tom-empathy467 diary. Due to privacy concerns (e.g., mental health and personal history), researchers from verified 468 institutions could email the managing institution to get access to the full data $(52,77)$. 
469

470

\section{1}

472

473

474

475

476

477

Table 1. The List of Self-Reported Measures in "COVID-19 Emotion Diary with Empathy and Theory-of-Mind Ground Truths Dataset" Used in This Study.

\begin{tabular}{lll}
\hline Variable Names & Number of Items & Item Scales \\
\hline Loneliness & 6 items & 1 to 4 \\
Extraversion & 2 items & 1 to 5 \\
Neuroticism & 2 items & 1 to 5 \\
Age & 1 item & 1 to 100 \\
Gender & 1 item & Male $=1$, Female $=0$ \\
Income Level & 1 item & 1 to 9 \\
Education Level & 1 item & 1 to 7 \\
Household Type & 1 item & 1 to 4 \\
Remote Working or Schooling & 1 item & Yes $=1$ or $\mathrm{No}=0$ \\
Social distancing & 1 item & Yes $=1$ or $\mathrm{No}=0$ \\
\hline
\end{tabular}

Income level: 1(less than 20k) to 9(90k to 100k). Education level: 1(middle school) to 6(doctorate degree), and 7(others). Household type: 1(living alone), 2(living with immediate family), 3(living with people other than immediate family), and 4(others).

Table 2. Hierarchical Regression Results on Age and Gender as a Predictor for Loneliness.

\begin{tabular}{|c|c|c|c|c|c|c|}
\hline & \multicolumn{3}{|c|}{ Model 1} & \multicolumn{3}{|c|}{ Model 2} \\
\hline & $\beta$ & $C I$ & $p$ & $\beta$ & $C I$ & $p$ \\
\hline Intercept & $>-0.001$ & $\begin{array}{c}-0.031, \\
0.031\end{array}$ & 1.000 & 0.041 & $\begin{array}{l}0.005 \\
0.076\end{array}$ & $0.024 *$ \\
\hline Age & 0.057 & $\begin{array}{l}0.026 \\
0.088\end{array}$ & $<0.001 *$ & 0.038 & $\begin{array}{l}0.001 \\
0.075\end{array}$ & $0.043 *$ \\
\hline Gender(1=female) & & & & -0.197 & $\begin{array}{r}-0.270,- \\
0.123\end{array}$ & $<0.001 *$ \\
\hline $\begin{array}{l}\text { Age - Gender }(1=\text { female }) \\
\text { Interaction }\end{array}$ & & & & 0.102 & $\begin{array}{c}0.033 \\
0.170\end{array}$ & $0.004 *$ \\
\hline
\end{tabular}
$p<0.001)$ and $0.012(F(3,4013)=15.13, p<0.001)$ respectively. The hypothesis test result for the 480 change in adjusted $R^{2}$ values between the two models is $F(2,4013)=16.09, p<0.001$. Coefficients $(\beta)$, $481 \quad p$-values $(p)$, and adjusted $R^{2}$ values are rounded to three decimal places. F-values are rounded to two 482 483 decimal places. $C I=95 \%$ Confidence Intervals of coefficients $(\beta){ }^{*} *$ statistically significant at $95 \%$ confidence level. 
Table 3. Hierarchical Regression Results on Loneliness with Multiple Risk Factors.

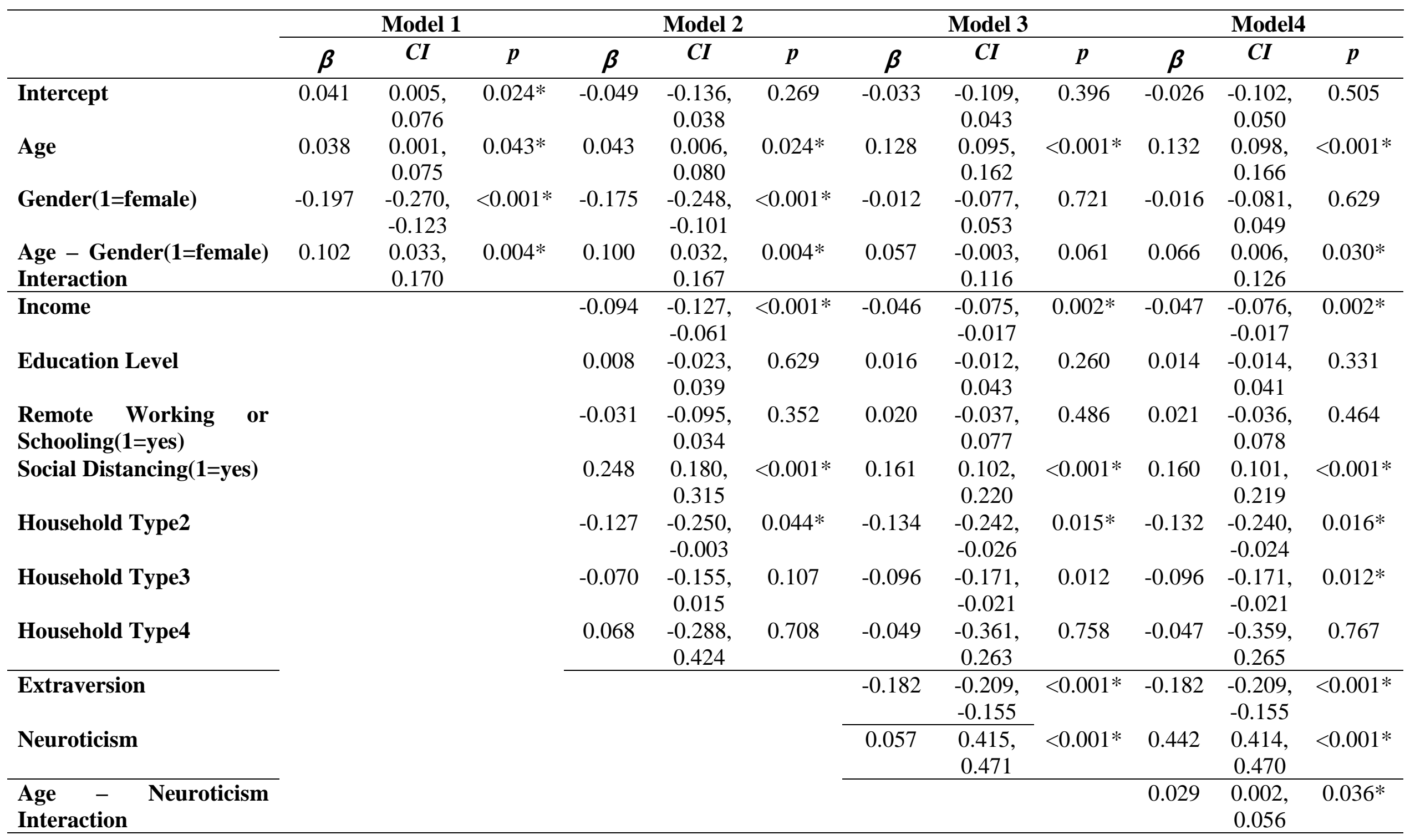

485 Household Type: 1(living alone), 2(living with immediate family), 3(living with people other than immediate family), and 4(others). All

486 continuous variables are standardized. Adjusted $R^{2}$ values for model 1, model 2, model 3, and model 4 are 0.011, 0.036, 0.259, and 0.257 
respectively. The hypothesis tests results for the change in adjusted $R^{2}$ values between the two models are $F(7,4006)=14.81, p<0.001$ for model 1 and model 2, $F(2,4004)=601.96, p<0.001$ for model 2 and model 3, and $F(3,4003)=403.11, p<0.001$ for model 2 and model 4 . 
Table 4. ANOVA Results for Differences in Loneliness by the Age Groups and Gender.

\begin{tabular}{lccccc}
\hline & $\boldsymbol{d} \boldsymbol{f}$ & Type III SS & $\boldsymbol{F}$ & $\boldsymbol{p}$ & $\begin{array}{c}\text { Partial } \\
\boldsymbol{\eta}^{2}\end{array}$ \\
\hline Intercept & 1 & 9824.00 & 19741.60 & $<0.001^{*}$ & \\
\hline Gender & 1 & 2.70 & 5.50 & $0.019^{*}$ & 0.001 \\
\hline Age Group & 4 & 23.20 & 11.60 & $<0.001^{*}$ & 0.012 \\
\hline Gender- Age Group Interaction & 4 & 10.20 & 5.10 & $<0.001^{*}$ & 0.005 \\
\hline Residuals & 4007 & 1994.10 & & & \\
\hline
\end{tabular}

492

493

494

495

496

497

Sum of squares $(S S)$ values and F-values $(F)$ are rounded to two decimal places. P-values $(p)$ and partial eta-squared $\left(\eta^{2}\right)$ values are rounded to three decimal places. $*=$ statistically significant at $95 \%$ confidence level.

Table 5. Planned Comparison Results for the Effect of the Age Groups and Gender on Loneliness.

\begin{tabular}{lcccc}
\hline & $\boldsymbol{\beta}$ & $\boldsymbol{S} \boldsymbol{E}$ & $\boldsymbol{t}$ & $\boldsymbol{p}$ \\
\hline Intercept & 2.293 & 0.016 & 140.50 & $<0.001^{*}$ \\
Gender & 0.038 & 0.016 & 2.35 & $0.019^{*}$ \\
Age Group1 & -0.023 & 0.006 & -3.83 & $<0.001^{*}$ \\
Age Group2 & 0.010 & 0.019 & 0.52 & 0.601 \\
Age Group3 & -0.074 & 0.017 & -4.69 & $<0.001^{*}$ \\
Age Group4 & 0.074 & 0.028 & 2.66 & $0.008^{*}$ \\
Gender- Age Group1 & 0.025 & 0.006 & 4.19 & $<0.001^{*}$ \\
$\begin{array}{l}\text { Interaction } \\
\text { Gender-Age Group2 }\end{array}$ & -0.028 & 0.017 & -1.68 & 0.094 \\
$\begin{array}{l}\text { Interaction } \\
\text { Gender-Age Group3 }\end{array}$ & 0.031 & 0.019 & 1.68 & 0.102 \\
$\begin{array}{l}\text { Interaction } \\
\text { Gender-Age Group4 }\end{array}$ & 0.007 & 0.028 & 0.24 & 0.814 \\
Interaction & & & & \\
\hline
\end{tabular}
their 40 s to $70 \mathrm{~s}$ ), age group2(individuals in their $60 \mathrm{~s}-70 \mathrm{~s}$ compared to those in their $30 \mathrm{~s}$ to $50 \mathrm{~s}$ ), age group3(individuals in their 20s compared to those in their 30s), and age group4(individuals in their 50140 s compared to those in their 50s) are contrasts of gender and age groups. The model was 502 statistically significant: $F(9,4007)=11.11, p<0.001$. * statistically significant at $95 \%$ confidence 503 level. 
Table 6. Unigrams that Greatly Varied in Frequencies by Gender among Participants in Their 50520 s and 30s.

\begin{tabular}{lcccccc}
\hline & \multicolumn{2}{c}{ 'child' } & \multicolumn{2}{c}{ 'confirmation' } & \multicolumn{2}{c}{ 'traveling' } \\
\cline { 2 - 7 } & \% & Ranks & \% & Ranks & \% & Ranks \\
\hline Female & 0.4756 & 14 & 0.4596 & 16 & 0.3742 & 23 \\
Male & 0.0023 & 56 & 0.3404 & 24 & 0.0025 & 44 \\
\hline
\end{tabular}

506

507

508

509

510

511

512 Table 8. Topics Related to Pets across the Female Age Groups.

Frequencies (\%) and ranks are calculated within the same gender-age group.

Table 7. Bigrams that that Greatly Varied in Frequencies by Gender among Participants in Their 20s and 30s.

\begin{tabular}{lcccccc}
\hline & \multicolumn{2}{c}{ 'delivery food' } & \multicolumn{2}{c}{ 'all day long' } & \multicolumn{2}{c}{$\begin{array}{c}\text { 'boyfriend' or } \\
\text { 'girlfriend' }\end{array}$} \\
\cline { 2 - 7 } & $\boldsymbol{\%}$ & Ranks & \% & Ranks & \% & Ranks \\
\hline Female & 0.0679 & 24 & 0.0861 & 16 & 0.1953 & 3 \\
Male & 0.0005 & 33 & 0.0565 & 29 & 0.3015 & 1 \\
\hline
\end{tabular}

Frequencies (\%) and ranks are calculated within the same gender-age group.

\begin{tabular}{ll}
\hline Age Groups & Topics \\
\hline $20 \mathrm{~s}$ & mom, sound, dad, dog, call, grandfather, cat, grandmother \\
$40 \mathrm{~s}$ & this time, trouble, cat, fact, one, study, event, moving \\
$50 \mathrm{~s}$ & workout, stroll, dog, stress, movie, a day, mood, start \\
$60 \mathrm{~s}-70 \mathrm{~s}$ & workout, mood, thought, stroll, dog, arrangement, park, apartment \\
\hline
\end{tabular}

513

514 Table 9. Topics Related to Mother across the Female Age Groups.

\begin{tabular}{ll}
\hline Age Groups & Topics \\
\hline $20 \mathrm{~s}$ & mom, sound, dad, dog, call, grandfather, cat, grandmother \\
$30 \mathrm{~s}$ & mom, workout, husband, a day, baby, start, mood, morning \\
$40 \mathrm{~s}$ & mom, mind, happiness, parents, travel, thought, husband, family \\
$50 \mathrm{~s}$ & mom, hospital, mind, family, parents, test, worry, father \\
$60 \mathrm{~s}-70 \mathrm{~s}$ & mind, thought, mom, alone, going to work, supermarket, extent, today \\
\hline
\end{tabular}


517 Table 10. Topics Related to Jobs and Working across the Age-Gender Groups.

\begin{tabular}{|c|c|c|}
\hline Age Groups & Gender & Topics \\
\hline \multirow[t]{4}{*}{$20 \mathrm{~s}$} & Female & $\begin{array}{l}\text { thought, emotion, mind, self/confidence, finding a position, } \\
\text { depression, happiness, situation }\end{array}$ \\
\hline & & $\begin{array}{l}\text { company, working from home, going to work, working, thought, } \\
\text { work, workplace, hospital }\end{array}$ \\
\hline & & $\begin{array}{l}\text { progress, interview, thought, non-contact, face, online, first, } \\
\text { meeting }\end{array}$ \\
\hline & Male & $\begin{array}{l}\text { work, part-time job, store, employee, going to work, boss, } \\
\text { problem, working }\end{array}$ \\
\hline \multirow[t]{3}{*}{$30 \mathrm{~s}$} & Female & $\begin{array}{l}\text { class, company, working from home, work, start, study, progress, } \\
\text { preparation }\end{array}$ \\
\hline & Male & $\begin{array}{l}\text { company, going to work, working, working from home, work, } \\
\text { workplace, employee, colleague }\end{array}$ \\
\hline & & $\begin{array}{l}\text { work, working from home, delivery, problem, situation, extent, } \\
\text { occasion, emotion }\end{array}$ \\
\hline \multirow[t]{3}{*}{$40 \mathrm{~s}$} & Female & $\begin{array}{l}\text { company, working from home, situation, call, work, husband, } \\
\text { hobby, workplace }\end{array}$ \\
\hline & Male & $\begin{array}{l}\text { company, work, working from home, progress, going to work, } \\
\text { one, colleague, meeting }\end{array}$ \\
\hline & & employee, annoyance, travel, office, eating, most, dinner, happiness \\
\hline $50 \mathrm{~s}$ & Male & $\begin{array}{l}\text { work, problem, visit, apartment, occasion, employee, progress, } \\
\text { management }\end{array}$ \\
\hline
\end{tabular}

Table 11. Topics Related to Spirituality among the Female Age Groups.

\begin{tabular}{ll} 
Age Groups & Topics \\
\hline $50 \mathrm{~s}$ & class, school, study, online, progress, church, student, situation \\
$60 \mathrm{~s}-70 \mathrm{~s}$ & $\begin{array}{l}\text { worship, preventative measure, church, last year, thought, vaccine, human, } \\
\text { government }\end{array}$
\end{tabular}


525 Table 12. Examples of Diaries Written by Females in Their 30s.

\begin{tabular}{ll}
\hline Topics & Texts from the Diaries \\
\hline Being a mother & $\begin{array}{l}\text { Due to the coronavirus, the daycare centers are temporarily closed as social } \\
\text { distancing measures have become stricter. I have to take care of my } \\
\text { newborn baby and a three-year-old child alone. }\end{array}$ \\
\cline { 2 - 3 } & $\begin{array}{l}\text { As a full-time stay-at-home mom, I had to help my daughter take an online } \\
\text { class when she couldn't go to school in person, and I had to prepare three } \\
\text { meals every day. One of the most painful things to do with the COVID-19 } \\
\text { is to prepare a meal for your family. }\end{array}$ \\
\hline $\begin{array}{l}\text { Losing contact with } \\
\text { friends who have } \\
\text { their kids }\end{array}$ & $\begin{array}{l}\text { She was a colleague I relied upon a lot, but I was very depressed to hear her } \\
\text { having to quit the job. It was because she had difficulties raising her } \\
\text { children due to coronavirus. I felt like I was left alone in the company. }\end{array}$ \\
\cline { 2 - 3 } & $\begin{array}{l}\text { My best friend is a mom raising a 5-year-old child. After the outbreak of } \\
\text { coronavirus, I can't go because I'm afraid that I might spread the virus to } \\
\text { them. Even if only one of the parents is infected with the virus, the whole } \\
\text { family will be quarantined, tested, and may become infected, so I can't visit } \\
\text { them anymore. }\end{array}$ \\
\hline
\end{tabular}




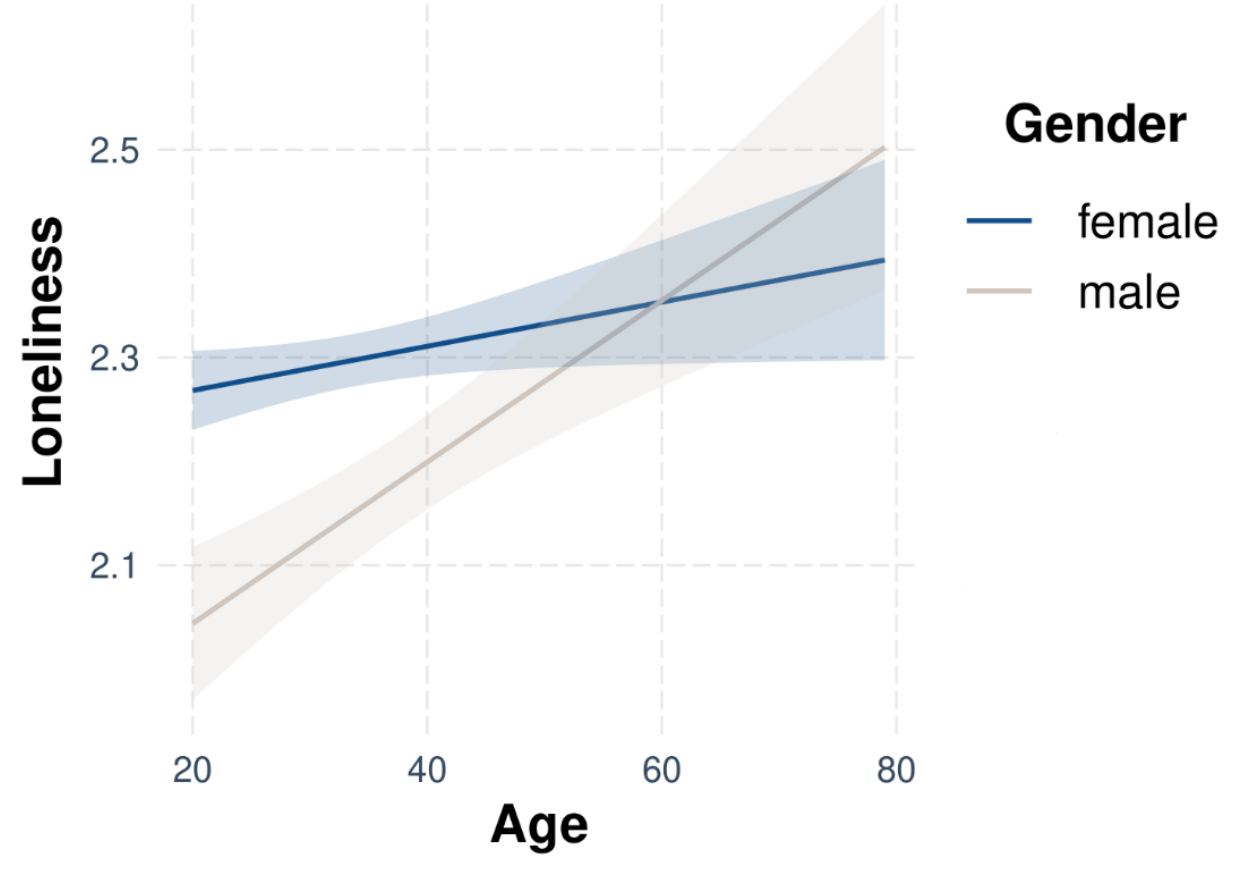

The blue line indicates the regression line of women, and the grey line indicates the regression line of men. Colored areas around the lines represent $95 \%$ prediction intervals.

Figure 2. Average Loneliness Scores by the Age Groups and Gender.

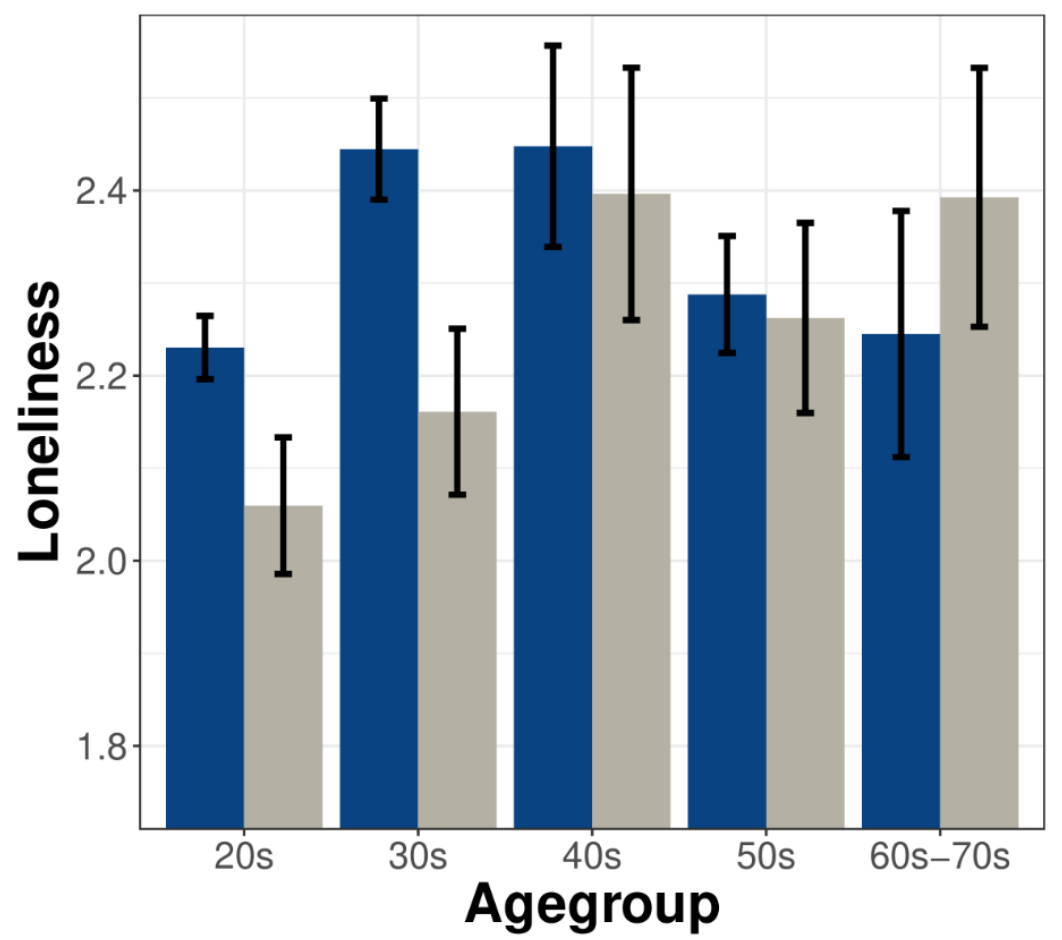

536 The blue bars indicate loneliness scores of women by age groups, and the grey bars indicate

537 loneliness scores of men by age groups. Error bars represent $95 \%$ confidence interval. 
Age-Gender Differences in Loneliness

540 1. Cacioppo JT, Patrick W. Loneliness: Human Nature and the Need for Social Connection.

541 New York: WW Norton \& Company (2008).

542 2. Cacioppo JT, Hughes ME, Waite LJ, Hawkley LC, Thisted RA. Loneliness as a Specific Risk 543 Factor for Depressive Symptoms: Cross-Sectional and Longitudinal Analyses. Psychol Aging (2006)

544 21(1):140-51. doi: 10.1037/0882-7974.21.1.140

545 3. Cacioppo JT, Hawkley LC, Ernst JM, Burleson M, Berntson GG, Nouriani B, et al.

546 Loneliness within a Nomological Net: An Evolutionary Perspective. J Res Pers (2006) 40(6):1054-

547 85. doi: 10.1016/j.jrp.2005.11.007

548 4. Elphinstone B. Identification of a Suitable Short-Form of the Ucla-Loneliness Scale. Aust 549 Psychol (2018) 53(2):107-15. doi: 10.1111/ap.12285

550 5. Szcześniak M, Bielecka G, Madej D, Pieńkowska E, Rodzeń W. The Role of Self-Esteem in 551 the Relationship between Loneliness and Life Satisfaction in Late Adulthood: Evidence from Poland. 552 Psychol Res Behav Manag (2020) 13:1201-12

553 6. Hawkley LC, Cacioppo JT. Loneliness Matters: A Theoretical and Empirical Review of

554 Consequences and Mechanisms. Ann Behav Med (2010) 40(2):218-27. doi: 10.1007/s12160-010-

$555 \quad 9210-8$

$5567 . \quad$ Valtorta NK, Kanaan M, Gilbody S, Ronzi S, Hanratty B. Loneliness and Social Isolation as 557 Risk Factors for Coronary Heart Disease and Stroke: Systematic Review and Meta-Analysis of 558 Longitudinal Observational Studies. Heart (2016) 102(13):1009-16. doi: 10.1136/heartjnl-2015559308790

$560 \quad$ 8. Holt-Lunstad J, Smith TB, Baker M, Harris T, Stephenson D. Loneliness and Social Isolation 561 as Risk Factors for Mortality: A Meta-Analytic Review. Perspect Psychol Sci (2015) 10(2):227-37.

562 doi: 10.1177/17456916145683529. Holt-Lunstad J, Smith TB, Layton JB. Social Relationships and 563 Mortality Risk: A Meta-Analytic Review. PLoS Med (2010) 7(7):e1000316. doi:

564 10.1371/journal.pmed.1000316

565 10. Abdellaoui A, Chen HY, Willemsen G, Ehli EA, Davies GE, Verweij KJ, et al. Associations 566 between Loneliness and Personality Are Mostly Driven by a Genetic Association with Neuroticism. $J$ 567 Pers (2019) 87(2):386-97. doi: 10.1111/jopy.12397

568 11. Luhmann M, Hawkley LC. Age Differences in Loneliness from Late Adolescence to Oldest 569 Old Age. Dev Psychol (2016) 52(6):943-59. doi: 10.1037/dev0000117

570 12. Pinquart M, Sörensen S. Risk Factors for Loneliness in Adulthood and Old Age--a Meta571 Analysis. New York: Nova Science Publishers (2003).

572 13. Wang B, Dong X. The Association between Personality and Loneliness: Findings from a 573 Community-Dwelling Chinese Aging Population. Gerontol Geriatr Med (2018)

574 4:2333721418778181. doi: 10.1177/2333721418778181

575 14. Killgore WD, Cloonan SA, Taylor EC, Dailey NS. Loneliness: A Signature Mental Health 576 Concern in the Era of Covid-19. Psychiatry Res (2020) 290:113117. doi:

$577 \quad$ 10.1016/j.psychres.2020.113117

578 15. Killgore WD, Cloonan SA, Taylor EC, Lucas DA, Dailey NS. Loneliness During the First 579 Half-Year of Covid-19 Lockdowns. Psychiatry Res (2020) 294:113551. doi:

$580 \quad 10.1016 /$ j.psychres.2020.113551 
16. Enea V, Eisenbeck N, Petrescu TC, Carreno DF. Perceived Impact of Quarantine on

582 Loneliness, Death Obsession, and Preoccupation with God: Predictors of Increased Fear of Covid-19.

583 Front Psychol (2021) 12:643977. doi: 10.3389/fpsyg.2021.643977

584 17. Rania N, Coppola I. Psychological Impact of the Lockdown in Italy Due to the Covid-19

585 Outbreak: Are There Gender Differences? Front Psychol (2021) 12:567470. doi:

586 10.3389/fpsyg.2021.567470

587 18. Luchetti M, Lee JH, Aschwanden D, Sesker A, Strickhouser JE, Terracciano A, et al. The

588 Trajectory of Loneliness in Response to Covid-19. Am Psychol (2020) 75(7):897-908. doi:

589 10.1037/amp0000690

590 19. Groarke JM, Berry E, Graham-Wisener L, McKenna-Plumley PE, McGlinchey E, Armour C.

591 Loneliness in the Uk During the Covid-19 Pandemic: Cross-Sectional Results from the Covid-19

592 Psychological Wellbeing Study. PloS One (2020) 15(9):e0239698. doi:

593 10.1371/journal.pone.0239698

594 20. Victor CR, Yang K. The Prevalence of Loneliness among Adults: A Case Study of the United 595 Kingdom. J Psychol (2012) 146(1-2):85-104. doi: 10.1080/00223980.2011.613875

596

597

21. Linehan T, Bottery S, Kaye A, Millar L, Sinclair D, Watson J. 2030 Vision: The Best and

Worst Futures for Older People in the Uk. London: Independent Age and International Longevity

598 Centre-UK (2014).

599 22. Banks J, Fancourt D, Xu X. Mental Health and the Covid-19 Pandemic. In: Helliwell JF,

600 Layard R, Sachs J, De Neve J-E, editors. World Happiness Report 2021. New York: Sustainable

601 Development Solutions Network (2021).

602 23. Banerjee D, Rai M. Social Isolation in Covid-19: The Impact of Loneliness. Int J Soc

603 Psychiatry (2020) 66(6):525-7. doi: 10.1177/0020764020922269

604 24. Cohen-Mansfield J, Hazan H, Lerman Y, Shalom V. Correlates and Predictors of Loneliness

605 in Older-Adults: A Review of Quantitative Results Informed by Qualitative Insights. Int

606 Psychogeriatr (2016) 28(4):557-76. doi: 10.1017/S1041610215001532

607 25. Shovestul B, Han J, Germine L, Dodell-Feder D. Risk Factors for Loneliness: The High

608 Relative Importance of Age Versus Other Factors. PloS One (2020) 15(2):e0229087. doi:

609 10.1371/journal.pone.0229087

610 26. Losada-Baltar A, Jiménez-Gonzalo L, Gallego-Alberto L, Pedroso-Chaparro MdS,

611 Fernandes-Pires J, Márquez-González M. "We Are Staying at Home.” Association of Self-

612 Perceptions of Aging, Personal and Family Resources, and Loneliness with Psychological Distress

613 During the Lock-Down Period of Covid-19. J Gerontol B Psychol Sci Soc Sci (2021) 76(2):e10-e6.

614 doi: 10.1093/geronb/gbaa048

615 27. Bornstein S, Magnus M. Feeling Lonelier During the Early Covid-19 Pandemic: A Cross-

616 Sectional Analysis of Adults Living in the United States. Psychiatry Res (2022) 316:114759. doi:

617 10.1016/j.psychres.2022.114759

618 28. Li LZ, Wang S. Prevalence and Predictors of General Psychiatric Disorders and Loneliness

619 During Covid-19 in the United Kingdom. Psychiatry Res (2020) 291:113267. doi:

$620 \quad 10.1016 /$ j.psychres.2020.113267

621 29. Jaspal R, Breakwell GM. Socio-Economic Inequalities in Social Network, Loneliness and

622 Mental Health During the Covid-19 Pandemic. Int J Soc Psychiatry (2022) 68(1):155-65. doi:

$623 \quad 10.1177 / 0020764020976694$ 
624 30. Fuller HR, Huseth-Zosel A. Older Adults' Loneliness in Early Covid-19 Social Distancing:

625 Implications of Rurality. J Gerontol B Psychol Sci Soc Sci (2022) 77(7):e100-e5. doi:

626 10.1093/geronb/gbab053

627 31. Choi EY, Farina MP, Wu Q, Ailshire J. Covid-19 Social Distancing Measures and Loneliness among Older Adults. J Gerontol B Psychol Sci Soc Sci (2022) 77(7):e167-e78. doi:

629 10.1093/geronb/gbab009

630 32. Borys S, Perlman D. Gender Differences in Loneliness. Pers Soc Psychol Bull (1985)

631 11(1):63-74. doi: 10.1177/0146167285111006

632 33. Russell D, Peplau LA, Cutrona CE. The Revised Ucla Loneliness Scale: Concurrent and

633 Discriminant Validity Evidence. J Pers Soc Psychol (1980) 39(3):472-80. doi: 10.1037/0022-

$634 \quad 3514.39 .3 .472$

635 34. Hansen T, Slagsvold B. Late-Life Loneliness in 11 European Countries: Results from the 636 Generations and Gender Survey. Soc Indic Res (2016) 129(1):445-64. doi: 10.1007/s11205-015637 1111-6

638 35. Yang F, Gu D. Predictors of Loneliness Incidence in Chinese Older Adults from a Life 639 Course Perspective: A National Longitudinal Study. Aging Ment Health (2020) 24(6):879-88. doi: $640 \quad 10.1080 / 13607863.2018 .1558174$

641 36. Zhou Z, Wang P, Fang Y. Loneliness and the Risk of Dementia among Older Chinese Adults: 642 Gender Differences. Aging Ment Health (2018) 22(4):519-25. doi: 10.1080/13607863.2016.1277976

643 37. Peng C, Burr JA, Han SH. Cognitive Function and Cognitive Decline among Older Rural 644 Chinese Adults: The Roles of Social Support, Pension Benefits, and Medical Insurance. Aging Ment 645 Health (2022):1-9. doi: 10.1080/13607863.2022.2088693

646 38. Tso IF, Park S. Alarming Levels of Psychiatric Symptoms and the Role of Loneliness During 647 the Covid-19 Epidemic: A Case Study of Hong Kong. Psychiatry Res (2020) 293:113423. doi:

$648 \quad 10.1016 /$ j.psychres.2020.113423

39. Xu J, Ou J, Luo S, Wang Z, Chang E, Novak C, et al. Perceived Social Support Protects Lonely People against Covid-19 Anxiety: A Three-Wave Longitudinal Study in China. Front Psychol (2020) 11:566965. doi: 10.3389/fpsyg.2020.566965

652 40. Bao L, Li W-T, Zhong B-L. Feelings of Loneliness and Mental Health Needs and Services 653 Utilization Among chinese Residents During the Covid-19 Epidemic. Glob. Health (2021) 17(1):51. 654 doi: 10.1186/s12992-021-00704-5

655 41. Jiao WY, Wang LN, Liu J, Fang SF, Jiao FY, Pettoello-Mantovani M, et al. Behavioral and 656 Emotional Disorders in Children During the Covid-19 Epidemic. J Pediatr (2020) 221:264-6. e1. doi: 657 10.1016/j.jpeds.2020.03.013

658 42. Lee H-S, Dean D, Baxter T, Griffith T, Park S. Deterioration of Mental Health Despite 659 Successful Control of the Covid-19 Pandemic in South Korea. Psychiatry Res (2021) 295:113570. 660 doi: $10.1016 /$ j.psychres.2020.113570

661 43. Yoon S, Choi S. Stress-Related to Covid-19, Anxiety, and Protective Factors among Middle662 Aged and Older Adults in the Largest Outbreak Areas in South Korea. Aging Ment Health (2022) 663 26(10):2090-9. doi: 10.1080/13607863.2021.2017850

664 44. Rodriguez AJ, Holleran SE, Mehl MR. Reading between the Lines: The Lay Assessment of 665 Subclinical Depression from Written Self-Descriptions. J. Pers. (2010) 78(2):575-98. doi:

666 10.1111/j.1467-6494.2010.00627.x 
45. Tov W, Ng KL, Lin H, Qiu L. Detecting Well-Being Via Computerized Content Analysis of

668 Brief Diary Entries. Psychol (2013) 25(4):1069-78. doi: 10.1037/a0033007

669 46. Hofmann SG, Moore PM, Gutner C, Weeks JW. Linguistic Correlates of Social Anxiety

670 Disorder. Cogn Emot (2012) 26(4):720-6. doi: 10.1080/02699931.2011.602048

671 47. Settanni M, Marengo D. Sharing Feelings Online: Studying Emotional Well-Being Via

672 Automated Text Analysis of Facebook Posts. Front Psychol (2015) 6:1045. doi:

$67310.3389 /$ fpsyg.2015.01045

674 48. Rodríguez-Arauz G, Ramírez-Esparza N, Pérez-Brena N, Boyd RL. Hablo Inglés Y Español:

675 Cultural Self-Schemas as a Function of Language. Front Psychol (2017) 8:885. doi:

$67610.3389 /$ fpsyg.2017.00885

677 49. Ireland ME, Pennebaker JW. Language Style Matching in Writing: Synchrony in Essays,

678 Correspondence, and Poetry. J Pers Soc Psychol (2010) 99(3):549-71. doi: 10.1037/a0020386

679 50. Boyd RL, Schwartz HA. Natural Language Analysis and the Psychology of Verbal Behavior:

680 The Past, Present, and Future States of the Field. J Lang Soc Psychol (2021) 40(1):21-41. doi:

$681 \quad 10.1177 / 0261927 X 20967028$

682 51. Li M. Application of Sentence-Level Text Analysis: The Role of Emotion in an Experimental

683 Learning Intervention. J Exp Soc Psychol (2022) 99:104278. doi: 10.1016/j.jesp.2021.104278

684 52. Lee YK, Jung Y, Lee I, Park JE, Hahn S. Building a Psychological Ground Truth Dataset 685 with Empathy and Theory-of-Mind During the Covid-19 Pandemic. Cogsci (2021) 43(43).

686 53. World Medical Association. Wma Declaration of Helsinki - Ethical Principles for Medical

687 Research Involving Human Subjects. (2014). https://www.wma.net/policies-post/wma-declaration-

688 of-helsinki-ethical-principles-for-medical-research-involving-human-subjects/ [Accessed August 15,

689 2022].

690 54. Korea National Institute for Bioethics Policy. Human Subjects Research.

691 https://www.irb.or.kr/menu01/RegulationTarget01.aspx [Accessed March 10, 2022].

692 55. Neto F. Loneliness among Portuguese Adolescents. Soc Behav Pers (1992) 20(1):15-21. doi:

$693 \quad 10.2224 /$ sbp.1992.20.1.15

694 56. Neto F. Psychometric Analysis of the Short-Form Ucla Loneliness Scale (Uls-6) in Older

695 Adults. Eur J Ageing (2014) 11(4):313-9. doi: 10.1007/s 10433-014-0312-1

696 57. Kim OS. Korean Version of the Revised Ucla Loneliness Scale: Reliability and Validity Test.

697 Kanho Hakhoe Chi (1997) 27(4):871-9.

698 58. Nettle D. Personality: What Makes You the Way You Are. Oxford, England: Oxford

699 University Press (2009).

700 59. Rammstedt B, Beierlein C. Can't We Make It Any Shorter? The Limits of Personality

701 Assessment and Ways to Overcome Them. J Individ Differ (2014) 35(4):212-20. doi: 10.1027/1614-

702 0001/a000141

703 60. Eisinga R, Te Grotenhuis M, Pelzer B. The Reliability of a Two-Item Scale: Pearson,

704 Cronbach, or Spearman-Brown? Int J Public Health (2013) 58(4):637-42. doi: 10.1007/s00038-012-

$705 \quad 0416-3$

706 61. Blei DM, Ng AY, Jordan MI. Latent Dirichlet Allocation. J Mach Learn Res (2003)

707 3(Jan):993-1022. 
708 62. Newman D, Asuncion A, Smyth P, Welling M. Distributed Algorithms for Topic Models. J

709 Mach Learn Res (2009) 10(62):1801-28.

710 63. bab2min, Fenstermacher D. Bab2min/Tomotopy: 0.10.0 (December 19, 2020). Zenodo

711 [Internet]. http://doi.org/10.5281/zenodo.4362292 [Accessed October 10, 2021].

712 64. Statistics Korea. Economically Active Population Survey in January 2021 (2021).

713 65. Azcona G, Bhatt A, Encarnacion J, Plazaola-Castaño J, Seck P, Staab S, et al. From Insights

714 to Action: Gender Equality in the Wake of Covid-19. New York: United Nations Entity for Gender

715 Equality and the Empowerment of Women (2020).

716 66. Dang H-AH, Nguyen CV. Gender Inequality During the Covid-19 Pandemic: Income,

717 Expenditure, Savings, and Job Loss. World Dev (2021) 140:105296. doi:

718 10.1016/j.worlddev.2020.105296

719 67. Almeida M, Shrestha AD, Stojanac D, Miller LJ. The Impact of the Covid-19 Pandemic on

720 Women's Mental Health. Arch Womens Ment Health (2020) 23(6):741-8. doi: 10.1007/s00737-020-

$721 \quad 01092-2$

722 68. Iyengar U, Jaiprakash B, Haitsuka H, Kim S. One Year into the Pandemic: A Systematic

723 Review of Perinatal Mental Health Outcomes During Covid-19. Front Psychiatry (2021) 12:845. doi:

724 10.3389/fpsyt.2021.674194

725 69. Igbokwe CC, Ejeh VJ, Agbaje OS, Umoke PIC, Iweama CN, Ozoemena EL. Prevalence of

726 Loneliness and Association with Depressive and Anxiety Symptoms among Retirees in Northcentral

727 Nigeria: A Cross-Sectional Study. BMC Geriatr (2020) 20(153). doi: 10.1186/s128777-020-01561-4

728

729

70. Guntuku SC, Schneider R, Pelullo A, Young J, Wong V, Ungar L, et al. Studying Expressions of Loneliness in Individuals Using Twitter: An Observational Study. BMJ open (2019)

730 9(11):e030355. doi: 10.1136/ bmjopen-2019-030355

731 71. Aglozo EY, Akotia CS, Osei-Tutu A, Annor F. Spirituality and Subjective Well-Being among 732 Ghanaian Older Adults: Optimism and Meaning in Life as Mediators. Aging Ment Health (2021)

733 25(2):306-15. doi: 10.1080/13607863.2019.1697203

734

735

736

737

738

739

72. Idler EL, Kasl SV. Religion among Disabled and Nondisabled Persons Ii: Attendance at Religious Services as a Predictor of the Course of Disability. J Gerontol B Psychol Sci Soc Sci (1997) 52B(6):S306-S16. doi: 10.1093/geronb/52B.6.S306

73. King M, Speck P, Thomas A. The Royal Free Interview for Spiritual and Religious Beliefs: Development and Validation of a Self-Report Version. Psychol Med (2001) 31(6):1015-23. doi: $10.1017 /$ S0033291701004160

740 74. Kirby SE, Coleman PG, Daley D. Spirituality and Well-Being in Frail and Nonfrail Older

741 Adults. J Gerontol B Psychol Sci Soc Sci (2004) 59(3):P123-P9. doi: 10.1093/gernob/59.3.P123

742 75. Zhang X, Chen Q. Towards an Understanding of the Decision Process of Solvers'

743 Participation in Crowdsourcing Contests for Problem Solving. Behav Inf Technol (2022)

744 41(12):2635-53. doi: 10.1080/0144929X.2021.1941258

745 76. Jung Y, Lee YK, Hahn S. Age and Gender Differences in Loneliness During the Covid-19:

746 Analyses on Large Cross-Sectional Surveys and Emotion Diaries. psyarxiv [Prepreint] (2022).

747 Available at: 10.31234/osf.io/2bdek. 
748 77. Lee YK, Jung Y, Lee I, Park JE, Hahn S. Data from: Covid-19 Emotion Diary with Empathy

749 and Theory-of-Mind Ground Truths Dataset. Github. (2021)

750 https://github.com/humanfactorspsych/covid19-tom-empathy-diary 